\title{
The Annual and Diurnal Heat-Exchange Cycles in Upper Layers of Soil ${ }^{1}$
}

\author{
J. E. Carson and H. Moses \\ Radiological Physics Division, Argonne National Laboratory
}

(Manuscript received 24 August 1962, in revised form 8 January 1963)

\begin{abstract}
Micrometeorologists have long recognized the role of the heat-storage capacity of the soil in such problems as the occurrence of frost and dew, forecasting of maximum and minimum temperatures, partition of solar energy between the soil and atmosphere, etc. Meteorologists are becoming increasingly aware of the importance of thermal properties of the soil on larger-scale processes such as the modification of air masses, formation of tornadoes, development of weather systems, and the general circulation.

The heat energy stored per unit area by the soil can be calculated if the temperature and heat capacity of the soil as functions of time and depth are known. In this investigation, the daily and annual cycles of soil temperature have been calculated from the soil temperature data collected by the Meteorology Group at the Argonne National Laboratory. Since data on soil moisture were not available, it was necessary to estimate the heat capacity of the soil from several spot measurements of the heat-capacity profiles and the rainfall record.
\end{abstract}

\section{Introduction}

Our atmosphere is essentially a heat engine whose driving mechanism is the differential warming between land and water areas, high and low latitudes, etc. The ultimate source of this energy is, of course, radiation from the sun. Most of this energy enters the atmosphere not by direct absorption of the sun's rays, but from an energy transformation at the air-surface interface, since the atmosphere is capable of retaining only a small fraction of the insolation passing through it. This energy enters the atmosphere in the form of sensible heat conducted from the earth's surface, long wave (infrared) radiation emitted at the surface, and latent heat of water vapor evaporated at the surface. The amount of solar energy reflected back toward space and the partition of the remaining insolation between the atmosphere and soil at the air-soil interface will depend in large measure on the characteristics of the soil and its cover. Thus any investigation of the energy budget of the atmosphere, on either a local (such as the diurnal heat exchange and the forecasting of temperature extremes and frost) or on a global scale (development of weather systems and the general circulation) must consider the thermal properties of the underlying medium as well as those of the air.

\section{Soil-air partitioning of solar energy}

During the daylight hours, the surface of the soil is heated by solar radiation to a temperature much higher

\footnotetext{
${ }^{1}$ This work was performed under the auspices of the U.S.
} Atomic Energy Commission. than that of the air above or the soil below; hence, heat energy (not used to change the phase of water in the soil) is conducted away from the surface into both the air and the deeper soil layers. The amount of heat energy absorbed at the earth's crust will depend on the intensity and duration of solar radiation and on the reflectivity and transmissivity of the surface. The amount of energy penetrating into the earth will depend upon the thermal properties of the soil, such as its conductivity, diffusivity, and specific heat, as well as such factors as the plant cover, exposure, and slope. Thus the soil plays an important role in the redistribution of solar radiation.

The influence of the ground on the layer of air immediately above is so large that two relatively new branches of meteorology, microclimatology and micrometeorology, have been developed to describe and to explain these interactions. Several investigations of the large-scale meteorological effects of this heat exchange have appeared in recent literature (Petterssen and Calabrese, 1959; Priestley ${ }^{2}$; Petterssen ${ }^{3}$; Budyko, 1958; Smagorinsky, 1953).

The surface layer of soil acts as a heat reservoirabsorbing part of the excess heat of midday and summer, and returning it during the night and winter to the atmosphere. This storage lessens the temperature extremes of the atmosphere and acts as a climatic stabilizer. During the day, when the soil surface is usually

2 Priestley, C. H. B., 1957 : The evolution of energy gain by the atmosphere through contact with the ground or ocean. Sci. Rept., No. 2, Contract AF 19(604)-2179, Univ. of Chicago, $56 \mathrm{pp}$.

${ }^{3}$ Petterssen, S., 1959: On the influence of heat exchanges on motion and weather systems. Sci. Rept. No. 10, Contract AF 19(604)2179 , Univ, of Chicago, $18 \mathrm{pp}$. 
much warmer than the air above, a steep lapse rate is formed in the atmosphere. This unstable condition leads to air convection, turbulence, and gustiness of the wind and, through these motions, to the removal of pollutants and water vapor from the air-soil interface. At night, the cool soil surface creates a stable lapse rate and may bring about a pollution problem of considerable importance, due to the lack of turbulence (and therefore reduced diffusing power of the atmosphere). On the other hand, if the air is sufficiently cooler than the soil, it may be possible for enough heat to flow upward to prevent or inhibit the formation of a nocturnal inversion and thus reduce the concentration of pollutants near the ground. Rider (1957) has shown that no inversion formed above a plot of bare soil on a clear night, while an inversion did form over an adjacent grass field. He attributes the lack of an inversion over the bare soil to the return of the heat stored in the soil during the day. This example underlines the importance of a grass cover to the microclimate. Kuhn et al. (1958) have given evidence that local "hot spots" in the surface thermal patterns are closely related to the place of formation of tornadoes. Saunders $(1960 \mathrm{a} ; 1960 \mathrm{~b})$ has shown that, with the arrival of a cloud sheet at night, the heat stored in the soil during the day is often sufficient to evaporate a water fog if the soil at the 2-inch depth is only $2 \mathrm{~F}$ warmer than the air above, or to remove frost. Petterssen $^{3}$ and Petterssen and Calabrese (1959) have recently pointed out the role of local heat sources, such as the Great Lakes in winter, on the motion and development of large weather systems.

\section{Measurement of soil heat flux}

Four techniques are available for estimating or measuring the flow of heat in the soil. A description of each follows:

a) The temperature gradient method. In principle, the flux of heat in the soil is given by

$$
S=-\lambda \frac{\partial T}{\partial z}
$$

where $S$ is the soil heat flux, $\lambda$ the thermal conductivity, and $\partial T / \partial z$ the vertical temperature gradient. Unfortunately, the method is difficult to use in soil where temperature gradients and the thermal conductivity vary rapidly with time and depth and are difficult to measure (Rider, 1955; McCulloch and Penman, 1956; de Vries, 1958). In addition, heat energy is transported through the soil by several other processes, such as by the diffusion of water vapor and by the movement of soil water and air.

b) The plate method. An instrument is used which consists of a thin plate of a material (usually plastic) whose thermal conductivity is known to be roughly that of soil. The thermal gradient across the plate is measured and the heat flow through the pla:e (and therefore also in the soil) may then be calculat d from $\mathrm{Eq}$ (1). The plate method offers two importan1 advantages over the other techniques listed, namely, (1) that the heat flux is measured directly, and (2) that several plates can be placed at various locations, giving $a$ more representative picture.

c) Heat-flow method. A technique for estimating heat storage within the soil may be obtilined by applying the theory of heat flow into a semi-infinite, homogeneous medium. The rate at which heat energy is conducted through a unit area of soil is given by $\mathrm{Eq}$ (1). This equation can be integrated with respect to time to obtain the amount of heat passing through the soil during the interval, if the thermal conductivity and temperature as functions of time and depth are known.

The annual temperature wave at any time $t$ at the soil surface can be approximated by the sine function

$$
T(0, t)=\tilde{T}+\Delta T_{0} \sin \left(\frac{i \pi}{P} t+\Phi_{0}\right)
$$

where $\Delta T_{0}$ is the amplitude of the surface temperature wave, $\bar{T}$ the average temperature, $P$ the period of the wave, and $\Phi_{0}$ a phase angle. Car son ${ }^{4}$ and others have shown that only one harmonic is reeded to describe the annual cycle of soil temperature. It it is further assumed that the soil is a semi-infinite, homogeneous medium, the temperature of any depth $z$ and time $t$ is (Ingersol et al., 1954)

$$
\begin{aligned}
T(z, t)=\bar{T}+\Delta T_{0} \exp (-z \sqrt{\pi / P \kappa}) \sin \\
\quad \times\left(\frac{2 \pi}{P} t+\Phi_{0}-z \sqrt{\pi / P_{\kappa}}\right),
\end{aligned}
$$

where $\kappa$ is the thermal diffusivity of the soil. This is the equation of the damped, lagging wave.

Substituting $\mathrm{Eq}$ (3) into (1) and integrating over one-half of a temperature cycle, one finds total heat flow per half cycle through the surface per unit area, $\Delta Q$, to be

$\Delta Q=\int_{0}^{P / 2} S d t=\Delta T_{0} \sqrt{2 P / \pi} \quad C \sqrt{\kappa}=\Delta T_{0} \sqrt{2 P C \lambda / \pi}$

where $\kappa$ is the thermal diffusivity a ad $C$ is the volumetric heat capacity (Ingersoll et al., 1954). The volumetric heat capacity is the product $\rho s$, vihere $\rho$ is the density and $s$ the specific heat. This equation shows that the heat storage per unit area is proportional to the square root of the product of the period and factor $(C \lambda)$, the conductive capacity of the mediu:n.

The combination of physical properties of any

${ }^{4}$ Carson, J. E., 1961 : Soil temperaturı and weather conditions. ANL-6470, Argonne National Laboritory, Argonne, Illinois, $244 \mathrm{pp}$. 
medium represented by $\left(C_{K^{\frac{1}{2}}}\right)=(\lambda C)^{\frac{1}{2}}$ has a critical role in all problems concerning heat transfer at a surface of discontinuity, such as the earth's surface. This term has been given various names. Priestley ${ }^{2}$ and Petterssen ${ }^{3}$ called this quantity the conductive capacity, Johnson (1954) called it the thermal property, and Haltiner and Martin (1957) used the term soil product. Recently, Businger and Buettner (1961) have reviewed the confused nomenclature for this parameter and have suggested that it be called the "thermal contact coefficient."

d) Temperature-integral method. The amount of heat energy stored (or lost) in a unit area of soil during a given time interval can be calculated if the temperature distribution within the soil at the beginning and end of the period and the heat capacity of the soil as a function of depth are known. The heat stored in a unit area of soil is

$$
\Delta Q=\int_{0}^{\infty} \rho s \Delta T d z=\int_{0}^{\infty} C \Delta T d z
$$

where $\Delta T$ is the temperature change during the interval, and $z$ increases with depth. The lower limit of integration is the soil surface, $z=0$; the upper limit in practice is such a depth $z$ that the temperature change during the time interval is zero. This technique of determining the heat flux at the earth's surface is known as the temperature-integral method and is the one most commonly used. It is relatively easy to obtain the timedepth temperature values, but it is much more difficult to obtain accurate measurements of the heat capacity (which in effect is determined by the soil moisture) as functions of time and depth. Another factor which limits the applicability of $\mathrm{Eq}(5)$ is the correction which must be applied for the storage in the layer between the soil surface and the upper temperature probe, since surface temperature is difficult to measure. An isothermal change of heat capacity caused by a change of soil moisture, constitutes a change of heat content of the soil profile.

The most complete measurements of soil heat flux were those conducted by several university groups during Project Great Plains in 1953 in O'Neill, Nebraska. Three of the four techniques listed above (all but the heat-flow equation) were used by the various teams in a side-by side comparison. The values obtained by the various investigators at $\mathrm{O}^{\prime} \mathrm{Neill}$ are not in good agreement (Lettau, 1957), indicating that better techniques for measuring the heat flux at the soil surface and in the soil are needed.

\section{Annual heat-exchange cycle}

A quantity of considerable interest to meteorologists and climatologists is the amount of heat energy stored in the soil during the summer and then returned to the atmosphere during the cooler season; this quantity can be computed from formula (5) if the annual range of soil temperature and heat capacity of the soil are known.

Fig. 1 is a plot of the extreme temperature profiles at the Argonne National Laboratory, Argonne, Illinois, based on decade (10-day) averages. The first 10 days of a month comprise the first decade, the next 10 the second, and the remaining days the third. The soil temperature values used in this report were collected at seven depths $(1,10,20,50,100,305$ and $884 \mathrm{~cm})$ by resistance thermometers and recorded continuously on a strip chart recorder. The data for the calendar years 1953, 1954 and 1955 were used, the period for which a complete set of observations on punch cards was available. Each data point in Fig. 1 represents the warmest (or coolest) 10-day average for that level.

In order to compute the heat storage per unit area from $\mathrm{Eq} \mathrm{(5)} \mathrm{allowance} \mathrm{must} \mathrm{be} \mathrm{made} \mathrm{for} \mathrm{the} \mathrm{exponential}$ decrease of the annual range of soil temperature with depth. The heat storage is given by the area enclosed by two extreme temperature profiles and the ground surface $(z=0)$, multiplied by the heat capacity per unit volume of the soil. Since the temperature change with seasons had not reached zero at the deepest level measured, it was necessary to extrapolate the curve downward and add this area to the region determined by the direct measurements.

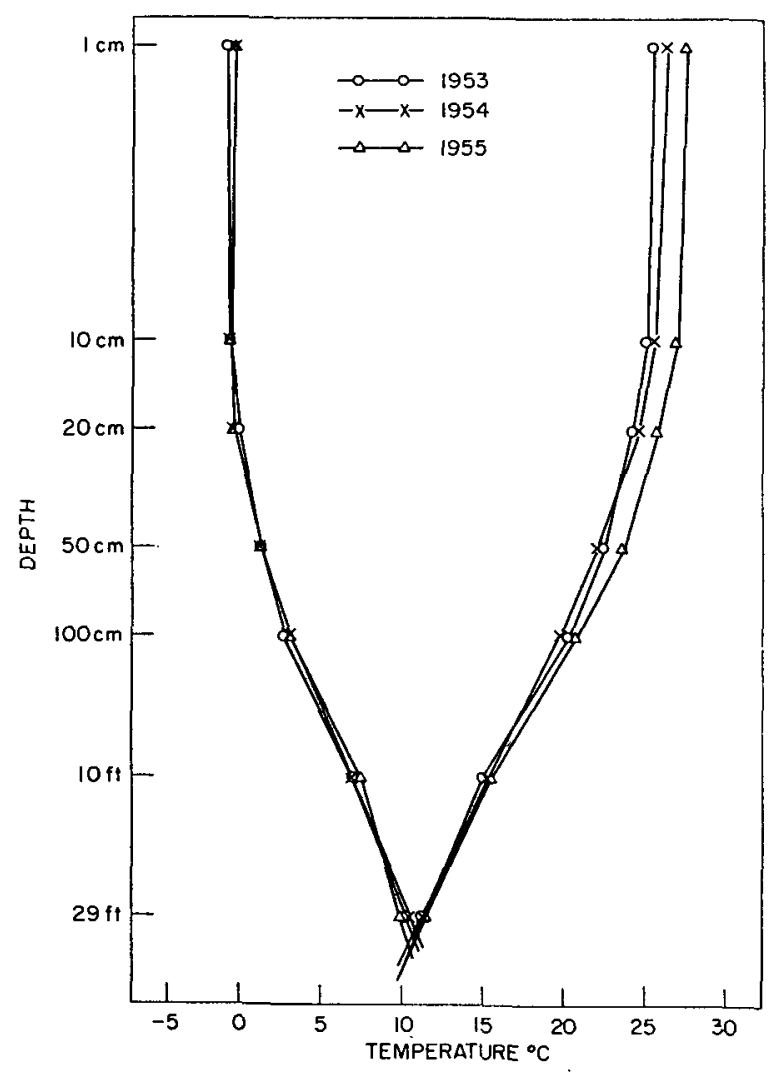

FIG. 1. Extremes of soil temperature at indicated depths on 10-day averages. 
The soil at Argonne is yellow clay of glacial origin with a cover of pasture grass. Kersten (1949) found that the volumetric heat capacity of silty clay loam varied from $0.44 \mathrm{cal} \mathrm{cm}^{-3} \mathrm{deg}^{-1}$ at low moisture contents to $0.70 \mathrm{cal} \mathrm{cm}^{-3} \mathrm{deg}^{-1}$ for clay pasture soil in England. Ingersol et al. (1954) reported a value of $0.59 \mathrm{cal} \mathrm{cm}^{-3}$ $\mathrm{deg}^{-1}$ for a sandy clay with 15 per cent moisture content.

Three determinations of the soil moisture, bulk density, and the specific heat of the soil at Argonne, under widely differing conditions of soil moisture, were made. The first set of samples was taken on 4 September 1959, after a prolonged hot, dry period. August 1959 was the second hottest August on record in Chicago, averaging $3.30 \mathrm{C}$ warmer than normal. After a heavy rain on 3 August, only small amounts of rain fell during the month prior to the date of the samples, as seen in Table 1 . As a result of the light rainfall, high temperature, and strong sunshine, the soil was extremely dry.

The second set of samples was taken on 27 October 1959, after the soil moisture content was recharged from the heavy rains of late September and October. Table 1 lists the daily rainfall totals in October prior to the sample. The third set was taken from under a deep blanket of snow on 19 February 1960. A storm on 9-10 February 1960, which began as snow and changed to freezing rain and then to sleet, left a total of 0.94 inch of precipitation and a snow cover of $18 \mathrm{~cm}$. The air temperature remained below the freezing point until the day of the sample, except for a short period on the 16th, when the air temperature reached a high of $+6.2 \mathrm{C}$. Ablation and compaction gradually reduced the snow cover to $10 \mathrm{~cm}$ on the date of the sample. Several inches of the soil were observed to be frozen when the sample was obtained. It is clear that this and the second series were taken when the soil moisture was near the maximum. The very dry series taken on 4 September 1959 was analyzed by the authors; the remaining two were analyzed by the Testing Service Corporation of Lombard, Ill.

A power-driven auger was used to drill a borehole $0.91 \mathrm{~m}$ in diameter to a depth of about $3 \mathrm{~m}$. Two soil samples at each of 6 depths were then collected for each

Table 1. Daily rainfall totals (inches) at Argonne National Laboratory in the period preceding soil samples (1959 data).

\begin{tabular}{cccc}
\hline \hline \multicolumn{2}{c}{ Sample A } & \multicolumn{2}{c}{ Sample B } \\
\hline 3 August & 1.38 & 2 October & 0.02 \\
7 August & 0.20 & 4 October & 0.36 \\
15 August & 0.23 & 5 October & 0.39 \\
16 August & 0.16 & 6 October & 1.06 \\
26 August & 0.06 & 8 October & 0.55 \\
28 August & 0.03 & 14 October & 0.14 \\
& & 23 October & 0.43 \\
& & 24 October & 0.43 \\
& & 26 October & 0.10 \\
Date of sample & Date of sample: \\
4 September & 27 October \\
\hline
\end{tabular}

determination. Thin-walled stainless steel cylinders (about $0.4 \mathrm{~m}$ long and $5.7 \mathrm{~cm}$ inside diameter) were used to obtain the first sample at each depth. These cylinders were driven horizontally into the soil with a hammer. The weight and volume of these relatively undisturbed samples were then used to determine the bulk density of the moist soil. After :he samples were removed from the tube and oven dried at $110 \mathrm{C}$ for 24 hours, the moisture content and dry density were computed. The second sample at each evel, used to measure the specific heat and moisture coitent of the soil, was collected from chunks of soil at the proper depth and kept in tightly sealed cans until analyzed.

The specific heats of the soil samples collected on 4 September 1959 were measured by a calorimetric technique. Each sample was carefully weighted and then chilled for about $72 \mathrm{hr}$ in a refrigerator to about $+2 \mathrm{C}$. The contents of the cans were then carefully placed in water slightly above rcom temperature and the contents well stirred; the method of mixtures was used to determine the specific hat of the sample of moist soil. The specific heat of the other two sets was obtained by means of a different procedure. The soil samples were first oven dried for 24 hours at about $110 \mathrm{C}$, powdered, and then placed in thin-walled copper containers. The specific heat of the dry soil in the containers was then measured in a jrecision calorimeter. This technique is certainly mora accurate than the method used on the first set. The :esults of these determinations are given in Table 2.

Several difficulties, in addition to those normally associated with measurement of :pecific heat, are encountered in attempting to measur e soil properties. One difficulty is the destructive nature of the test, which precludes repeated observations in the same location. It is impossible to replace the soil exartly and it is therefore necessary to take each set of samples at a different location. The presence of a borehole also will alter the moisture-temperature distributions in the surrounding soil. Hence, samples should not be sbtained near permanent soil-temperature measuring sites. The boreholes used in this report were located approximately $15 \mathrm{~m}$ to the east of the Thermohms used to neasure soil temperature. Another problem associated with soils is the lack of horizontal homogeneity of the :soil; this condition is pronounced in a glacial soil such as is present at Argonne. Thus, the samples used in this study may or may not be representative of the soil profile at the temperature-measuring site. Another unavoidable difficulty is the change in compaction of the soil when the stainless steel cylinders are drives into the soil. Considerable force is necessary to insert and remove these tubes from the clay soil. This, of course, changes the density of the sample as compared with the undisturbed values. The samples were quickly placed in tight containers to keep the loss of soil moisture, due to evaporation, to a minimum. 
Table 2. Thermal properties of the soil.

\begin{tabular}{|c|c|c|c|c|c|c|c|c|c|c|c|c|c|}
\hline \multirow{2}{*}{$\begin{array}{l}\text { Depth, } \\
\text { inches }\end{array}$} & \multicolumn{5}{|c|}{$\begin{array}{l}\text { Moisture content, } \\
\% \text { dry weight }\end{array}$} & \multicolumn{4}{|c|}{$\begin{array}{l}\text { Dry specific heat, } \\
\text { cal g }^{-1}(\text { deg C })^{-1}\end{array}$} & \multicolumn{4}{|c|}{$\begin{array}{l}\text { Moist specific heat, } \\
\text { cal g }^{-1}(\operatorname{deg} C)^{-1}\end{array}$} \\
\hline & $\mathrm{B}$ & $\mathrm{C}$ & D & E & $\mathrm{F}$ & $A^{*}$ & D & $\mathbf{E}$ & F & A & $\mathrm{D}^{*}$ & $\mathrm{E}^{*}$ & $\mathrm{~F}^{*}$ \\
\hline 4 & 10.6 & 26.8 & 22.6 & 27.4 & 22.6 & 0.219 & 0.205 & 0.216 & 0.206 & 0.294 & 0.352 & 0.352 & 0.385 \\
\hline 8 & 9.9 & 18.7 & 19.3 & 22.1 & 21.7 & 0.191 & 0.198 & 0.210 & 0.208 & 0.264 & 0.328 & 0.349 & 0.353 \\
\hline 20 & 17.8 & 23.8 & 22.8 & 27.5 & 28.0 & 0.224 & 0.211 & 0.211 & 0.210 & 0.341 & 0.357 & 0.383 & 0.396 \\
\hline 36 & 14.3 & 12.6 & 14.4 & 19.9 & 16.9 & 0.207 & 0.202 & 0.203 & 0.232 & 0.306 & 0.302 & 0.343 & 0.335 \\
\hline 69 & 16.4 & 16.0 & 15.8 & 16.4 & 16.9 & 0.214 & 0.208 & 0.200 & 0.218 & 0.325 & 0.316 & 0.331 & 0.313 \\
\hline \multirow[t]{9}{*}{108} & 15.5 & 14.5 & 15.1 & 17.6 & 17.3 & 0.215 & 0.216 & 0.201 & 0.194 & 0.321 & 0.319 & 0.313 & 0.321 \\
\hline & \multirow{2}{*}{\multicolumn{2}{|c|}{$\begin{array}{l}\text { Depth, } \\
\text { inches }\end{array}$}} & \multicolumn{3}{|c|}{$\begin{array}{l}\text { Dry bulk density, } \\
\mathrm{g} \mathrm{cm}^{-3}\end{array}$} & \multicolumn{3}{|c|}{$\begin{array}{l}\text { Moist builk density } \\
\mathrm{g} \mathrm{cm}^{-3}\end{array}$} & \multicolumn{4}{|c|}{$\begin{array}{l}\text { Volumetric heat capacity, } \\
\text { cal } \mathrm{cm}^{-3}\left(\operatorname{deg} \mathrm{C}^{-1}\right.\end{array}$} & \\
\hline & & & $\mathrm{B}^{* *}$ & C & $\mathrm{E}$ & $\mathrm{B}$ & C & E & $\mathrm{B}$ & $\mathrm{C}^{* * *}$ & $\mathrm{E}$ & $\mathrm{F} \dagger$ & \\
\hline & \multicolumn{2}{|c|}{4} & 1.28 & 1.45 & 1.52 & 1.42 & 1.83 & 1.87 & 0.417 & 0.644 & 0.658 & 0.672 & \\
\hline & & & 1.51 & 1.67 & 1.59 & 1.66 & 1.98 & \multirow[t]{2}{*}{1.94} & 0.438 & 0.649 & 0.677 & 0.679 & \\
\hline & & & 1.55 & 1.48 & 1.47 & 1.83 & 1.83 & & 0.624 & 0.653 & 0.720 & \multirow{2}{*}{$\begin{array}{l}0.722 \\
0.677\end{array}$} & \\
\hline & & & 1.77 & 1.78 & 1.82 & 2.02 & 2.00 & $\begin{array}{l}1.88 \\
2.12\end{array}$ & 0.618 & 0.604 & 0.727 & & \\
\hline & & & 1.80 & 1.70 & 1.68 & 2.10 & 1.97 & 1.97 & 0.683 & 0.622 & 0.652 & 0.620 & \\
\hline & & & 1.82 & 1.82 & 1.82 & 2.10 & 2.09 & 2.14 & 0.674 & 0.699 & 0.670 & 0.681 & \\
\hline
\end{tabular}

$\begin{array}{cc}\text { Sample } & \text { Date } \\ \text { A } & \text { 4 September 1959, can } \\ \text { B } & \text { 4 September 1959, tube } \\ \text { C } & \text { 27 October } 1959, \text { tube } \\ \text { D } & \text { 27 October 1959, can } \\ \text { E } & \text { 19 February 1960, tube } \\ \text { F } & \text { 19 February 1960, can }\end{array}$

The specific heat of moist soils, depends upon the specific heat of the dry matter, $s_{d}$, and the moisture content. Repeated experiments have shown that most mineral soils, such as gravel, clay, silt, and sand, have approximately the same dry specific heat. Most of the values fall in the range from 0.18 to $0.22 \mathrm{cal} \mathrm{gm}^{-1} \mathrm{deg}^{-1}$ (Patten, 1909; Kersten, 1949; Johnston, 1937; Carter, 1951; Smith, 1939; Portman ${ }^{5}$ ). Kersten found that the dry specific heat decreases 11 per cent for a $100 \mathrm{~F}$ drop in temperature. The value of the specific heat of moist soils is given by

$$
s=\frac{s_{d}+s_{w} W}{1+W}=\frac{s_{d}+W}{1+W}
$$

where $W$ is the moisture content of the soil (expressed as the mass of water per unit mass of the dry soil) and $s_{w}$ is the specific heat of water. This formula follows since the specific heat of water is by definition, unity.

The bulk density of natural soil depends upon the dry density and the moisture content. The dry density depends upon the nature of the soil material and the degree of compaction. The bulk density of moist soil is given by the relation

$$
\rho=\rho_{d}(1+W),
$$

${ }^{5}$ Portman, D. J., 1955: An investigation of the theory and measurement of heat transfer in natural soil. Unpublished Ph.D. dissertation, Johns Hopkins Univ., $218 \mathrm{pp}$.
* Computed from the formula $s=\left(s_{d}+W\right) /(1+W)$, where $s$ and $s_{d}$ are the specific heats of wet and dried soil samples, and $W$ is the moisture content of the soil.

** Compiled from the formula $\rho=\rho_{d}(1+W)$, where $\rho_{d}$ and $\rho$ are the dry and moist bulk densities.

*** The specific heat of sample $\mathrm{C}$ was not measured. The specific heat of dry soil for sample $D$ was used in the calculation.

T The bulk density of sample $F$ was not measured. The value listed in this column was calculated from the dry bulk density values from sample $E$ and the moisture and specific heat values of sample F.

where $\rho_{d}$ is the dry density and $W$ is the moisture content, as before. As can be seen from these equations, the value of the volumetric heat capacity of moist soil, $C$, is therefore

$$
C=\rho s=\rho_{d}\left(s_{d}+W\right) .
$$

Thus the problem of measuring the heat capacity of soil is essentially that of measuring its moisture content.

The results of these calculations are listed in Table 2. There is some scatter in the values, but the values are in agreement with the findings of other investigators.

A realistic set of values for the volumetric heat capacity profile, namely, 0.64 from surface to $20 \mathrm{~cm}$, 0.66 from 20 to $305 \mathrm{~cm}$, and 0.68 from there to the depth where the annual temperature cycle is completely damped out, gives a value of the annual heat storage of $4650 \mathrm{cal} \mathrm{cm}^{-2}$ for $1953 ; 4480 \mathrm{cal} \mathrm{cm}^{-2}$ for 1954 ; and $4980 \mathrm{cal} \mathrm{cm}^{-2}$ for 1955 (assuming that the annual range of temperature in the soil above $1 \mathrm{~cm}$ was the same as at $1 \mathrm{~cm}$ ). The validity of these numbers, of course, depends to a considerable degree on the accuracy of the assumed values of $C$. It is quite possible that these estimates are in error by as much as 20 per cent.

The use of 10-day average temperatures, rather than extreme daily values, slightly lowers the area enclosed in Fig. 1, and thus produces a lower value of the heat storage. The computed value also does not include the amount of heat energy needed in spring to melt the 
frozen soil and released in the fall when the soil freezes. If it is assumed that the soil at Argonne freezes to a depth of about $25 \mathrm{~cm}$ (the depth of intersection of the $0 \mathrm{C}$ isotherm and the left temperature profile of Fig. 1) and has a typical winter soil-moisture content, an additional heat exchange of 400 to $500 \mathrm{cal} \mathrm{cm}^{-2}$ is indicated.

This calculation of the heat stored under a unit area of soil from the time of the minimum in winter to the maximum temperature profile in summer, gives a value considerably greater than the values given by Kühl (1907) and Schubert (1904). Kühl stated that the heat storage at latitudes above 35 degrees is only 1500 to $2500 \mathrm{cal} \mathrm{cm}^{-2}$. For a sandy soil at Eberswalde, near Berlin, Schubert found that the annual heat storage in grass-covered soil was $1850 \mathrm{cal} \mathrm{cm}^{-2}$. Hann and Süring (1943) found that the annual heat storage in Lake Geneva was $37,000 \mathrm{cal} \mathrm{cm}^{-2}$, showing the much smaller heat storage capacity of land as compared with lakes and water bodies under somewhat similar climatic conditions.

Another estimate of the amount of heat stored in the soil during the annual cycle may be obtained by means of $\mathrm{Eq}(4)$.

Using representative values of $\kappa$ and $C\left[0.005 \mathrm{~cm}^{2} \mathrm{sec}^{-1}\right.$ and $0.66 \mathrm{cal} \mathrm{cm}^{-3} \mathrm{deg}^{-1}$, respectively] for the soil at Argonne (Carson, 1963) and the observed 1-cm amplitude, this equation gives an annual heat storage of $5500 \mathrm{cal} \mathrm{cm}^{-2}$ in 1953,5620 in 1954, and 5840 in 1955. Since the amplitude of the temperature wave at $1 \mathrm{~cm}$ is less than that at the surface, this computation underestimates the heat storage by less than one per cent.

In deriving $\mathrm{Eq}(4)$, it was assumed that heat is transported in the soil by molecular conduction only, and that the soil is a homogeneous, semi-infinite medium. Since these assumptions are not valid in the soil, one cannot expect this calculation to give the same value for the heat storage as the more exact $\mathrm{Eq}(5)$.

\section{Annual heat-exchange cycles}

Eq (5) has been used to estimate the annual and diurnal cycles of heat exchange in the soil at Argonne. In order to make this calculation, it was necessary to assume reasonable values for the volumetric heat capacity since adequate data about soil moisture are not available for the period of record. The values of heat capacity listed in Table 2 are close to the extreme limits of this parameter, since they were obtained under conditions of very dry or very wet soil. With these values and the rainfall record, a representative value of $C$ was estimated each month at each of the levels where the soil temperature was measured. It was further assumed that the values of $C$ at 305 and $884 \mathrm{~cm}$ were constant through the year and equal to 0.70 and $0.71 \mathrm{cal} \mathrm{cm}^{-3}$ $\operatorname{deg}^{-1}$, respectively. It was also necessary to assume a linear variation of temperature change and heat capacity between levels.
The programs for these calculations were prepared by Mr. J. S. Dick of the Applied Mathematics Division of the Argonne National Laboratory for the GEORGE electronic computer. Two slightly different techniques were used to estimate the annual zycle of heat storage in the soil. In the first program, the value of $\Delta T$ used was the change of average monthly temperature from one month to the next at each of the seven levels. Fig. 2 is a plot of the vertical emperature profiles, based on monthly averages, for wach month in 1953, with the temperature data for the $384-\mathrm{cm}$ level omitted for clarity. The slopes of these profiles determined the direction and magnitude of the vertical heat flux. The net amount of heat entering or leitving the deeper soil layers during a month is given by the area enclosed by the two temperature profiles, co:rected for the heat capacity of the various soil layers.

The mean monthly temperature can be considered as typical of conditions in midmonth. Thus, for example, the area in Fig. 2 bounded by the temperature profiles for May and June and the soil surface represents the heat gained by the soil per unit ar a between mid-May

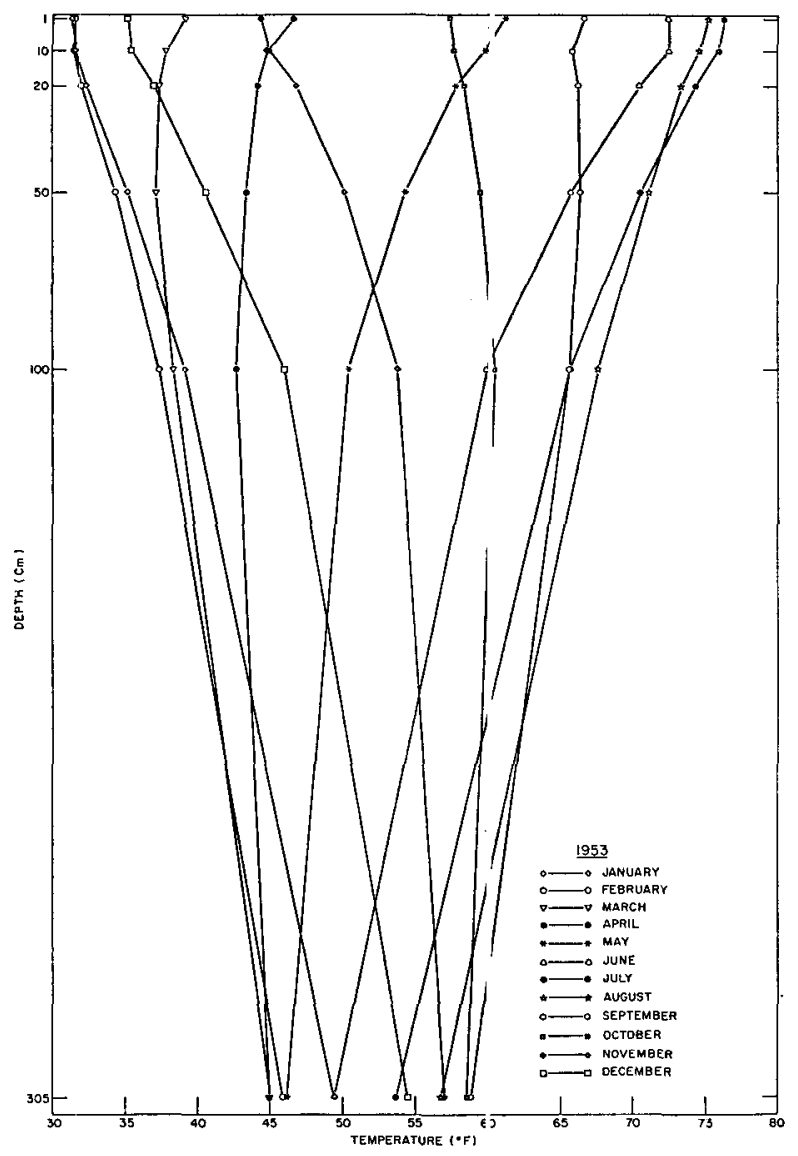

FIg. 2. Soil-temperature profiles for 1953 based on monthly averajres. 
and mid-June. In Table 3, the data represent the average amount of heat gained or lost by a unit area per day for the period ending in the middle of the month listed.

Since most climatological summaries are based on the calendar month, the interval from the middle of one month to the middle of the next is not the most convenient. Tables 4 and 5 and Fig. 3 were constructed by means of $\mathrm{Eq}(5)$, the assumed heat capacity values, and the temperature change from the last day of one month to the last day of the following month. In both Tables 3 and 4 it can be seen that there is little heat exchanged between air and soil in March and September, when the vertical temperature gradients in the soil were near zero (see Fig. 2). The maximum fluxes to and from the soil come at or just before the time of maximum and minimum insolation. Abnormally warm and cold months are reflected in the soil heat flux pattern.

Fig. 3 shows the annual march of soil heat exchange. This figure is based on 3-yr averages for each month. This curve is quite similar to the one shown by Pearce and Gold (1959) for the soil heat flux at Ottawa, Canada, as measured by a heat-flux transducer.

Another estimate of the amplitude of the annual heat storage is given in Table 5. This table is based on the data from Tables 3 and 4 and represents the total heat gained (or lost) by the soil during the 6 months of heat gains (or losses). These values underestimate the amplitude of the annual heat exchange, due to the averaging process. The values of heat storage indicated in this table are about 20 per cent smaller than those determined in the calculation based on annual temperature ranges. Table 5 also shows that the heat gains and losses computed using the data for the last day of the month are larger than those computed from monthly averages. The net accumulation during a given year would seem to indicate that the soil became warmer in 1953 and cooler in 1954 and 1955. Since the net values are small, a more probable explanation is the unrepresentativeness of assumed values of heat capacity.

\section{Diurnal heat-exchange cycles}

The magnitude of the daily heat exchange can also be estimated from $\mathrm{Eq}$ (5). For this calculation, the average daily range of soil temperature at $1,10,20$, and $50 \mathrm{~cm}$ for each month of the 3-yr period was used to estimate the daily change in temperatures. The values of $C$ used to prepare Tables 3 and 4 were also used in this calculation. The results of this calculation are given in Table 6 . Each value in the table is the average amount of heat energy in calories entering a unit area of soil during the warming portion of the daily cycle and then returning to the atmosphere during the cooling portion.

The data in Table 6 may be compared with the work of Suomi, Portman, and others, who measured the daily exchange of heat between the soil and atmosphere on clear days in late summer during the "Project Great
TABLE 3. Average amount of heat energy $\left(\mathrm{cal} \mathrm{cm}^{-2}\right.$ day $^{-1}$ ) gained or lost per day by the entire soil profile.*

\begin{tabular}{lccc}
\hline \hline & & Year & \\
Month & 1953 & 1954 & 1955 \\
\hline January & -23 & -30 & -25 \\
February & -18 & -17 & -18 \\
March & -2 & -4 & -7 \\
April & +10 & +13 & +16 \\
May & +26 & +25 & +33 \\
June & +39 & +38 & +28 \\
July & +30 & +32 & +32 \\
August & +18 & +18 & +24 \\
September & +4 & +3 & 0 \\
October & -14 & -13 & -18 \\
November & -26 & -34 & -34 \\
December & -31 & -34 & -35
\end{tabular}

* Data based on the change of average monthly soil temperature.

TABLE 4. Average amount of heat energy $\left(\mathrm{cal} \mathrm{cm}^{-2} \mathrm{day}^{-1}\right)$ gained or lost per day by the entire soil profile.*

\begin{tabular}{lccc}
\hline & & Year & \\
Month & 1953 & 1954 & 1955 \\
\hline January & -17 & -23 & -20 \\
February & -14 & -6 & -10 \\
March & +5 & -7 & -4 \\
April & +15 & +34 & +33 \\
May & +39 & +23 & +31 \\
June & +40 & +45 & +29 \\
July & +22 & +23 & +33 \\
August & +14 & +10 & +10 \\
September & -10 & -5 & -8 \\
October & -20 & -30 & -25 \\
November & -30 & -31 & -40 \\
December & -34 & -33 & -32
\end{tabular}

* Data based on the change of soil temperature on the last day of each month.

TABLE 5. Heat gains and losses $\left(\mathrm{cal} \mathrm{cm}^{-2}\right)$ from the entire soil profile during the course of a year.*

\begin{tabular}{lccccccc}
\hline \hline & \multicolumn{4}{c}{ Average monthly } & \multicolumn{4}{c}{ Last day of month } \\
& 1953 & 1954 & 1955 & 1953 & 1954 & 1955 \\
& & & & & & \\
& & & & & \\
Gains & +3886 & +3869 & +4004 & +4165 & +4111 & +4177 \\
Losses & -3455 & -4020 & -4165 & -3807 & -4142 & -4236 \\
Net & +431 & -151 & -161 & +358 & -31 & - \\
\hline
\end{tabular}

* Data based on Tables 3 and 4 and represent the total amount of heat entering the soil in summer and leaving in winter. For comparison, the annual heat exchange estimated from extreme decade temperature profiles for 1953 is $4650 \mathrm{cal} \mathrm{cm}^{-2}$; for 1954 , 4480 ; and for 1955,4980 .

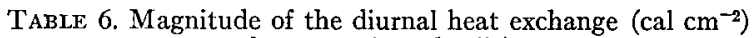
between air and soil.*

\begin{tabular}{llllllll}
\hline \hline $\mathrm{J}$ & 12 & $\mathrm{~A}$ & 75 & $\mathrm{~J}$ & 90 & $\mathrm{O}$ & 58 \\
$\mathrm{~F}$ & 15 & $\mathrm{M}$ & 87 & $\mathrm{~A}$ & 78 & $\mathrm{~N}$ & 41 \\
$\mathrm{M}$ & 46 & $\mathrm{~J}$ & 85 & $\mathrm{~S}$ & 68 & $\mathrm{D}$ & 16
\end{tabular}

* Data based on the average daily range of soil temperature'for each month for the 3-yr period 1953-1955. 
Plains" at O'Neill, Nebraska (Lettau, 1957); in some cases, smaller values -60 to $70 \mathrm{cal} \mathrm{cm}^{-2}$-were reported.

The vertical temperature profiles in the soil during the course of a typical sunny day in summer (27 June 1955) are shown in Fig. 4. The strong temperature gradient observed in the upper soil was directed downwards during the day. During the night, the soil surface cooled rapidly and the gradient was upwards above $20 \mathrm{~cm}$. The

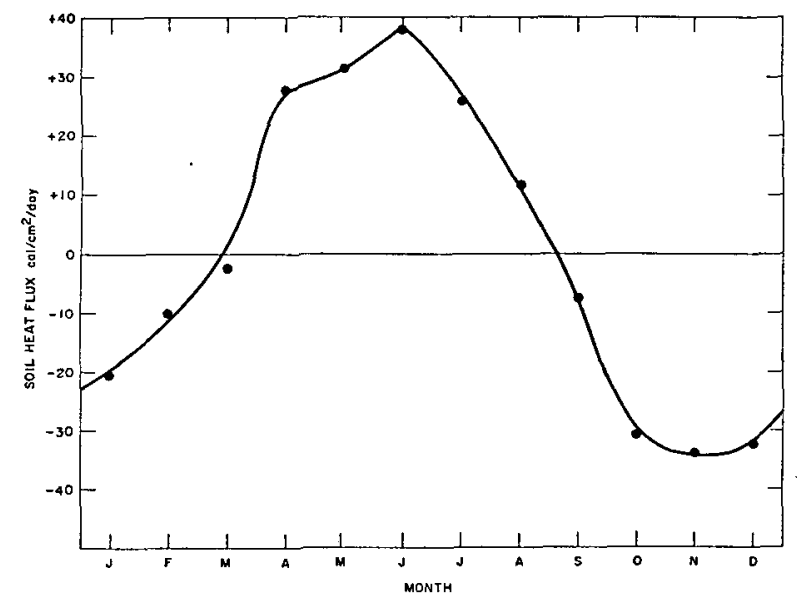

Frg. 3. Annual cycle of heat flux in soil. Data based on 3-yr period, 1953-55, and represent the change of heat storage in the soil per day from the surface to $29 \mathrm{ft}$ during the month indicated. strong temperature gradient from 50 to $100 \mathrm{~cm}$ indicates a-strong flux of heat into the deeper soil levels during the entire day.

The daily cycle of heat exchang: between the air and soil was computed from Eq (5), the observed average monthly hour-to-hour temperatu:e changes and the assumed values of $C$.

Fig. 5 shows the results of this calculation for alternate months in 1953. The heat-flux patterns are quite similar to those for the net radiation. The maximum flux into the soil is reached at or :ust before noon (the time of maximum net and solar radiation), but several hours before the maxima of the air- and soil-surface temperatures. The flux is negative from about $1600 \mathrm{CST}$ and remains negative for several hours after sunrise. The heating part of the cycle is thus much shorter and more intense than the cooling. The shape of the curves compare quite favorably with acti1al measured values, such as those reported by Lettau (1957).

\section{Conclusions}

The amount of heat energy stored (or lost) per unit area of soil during a given time interval can be calculated if the temperature distribution within the soil at the beginning and end of the perio 1 and the volumetric heat capacity of the soil are known. The estimates of heat storage given in this paper may be in error by

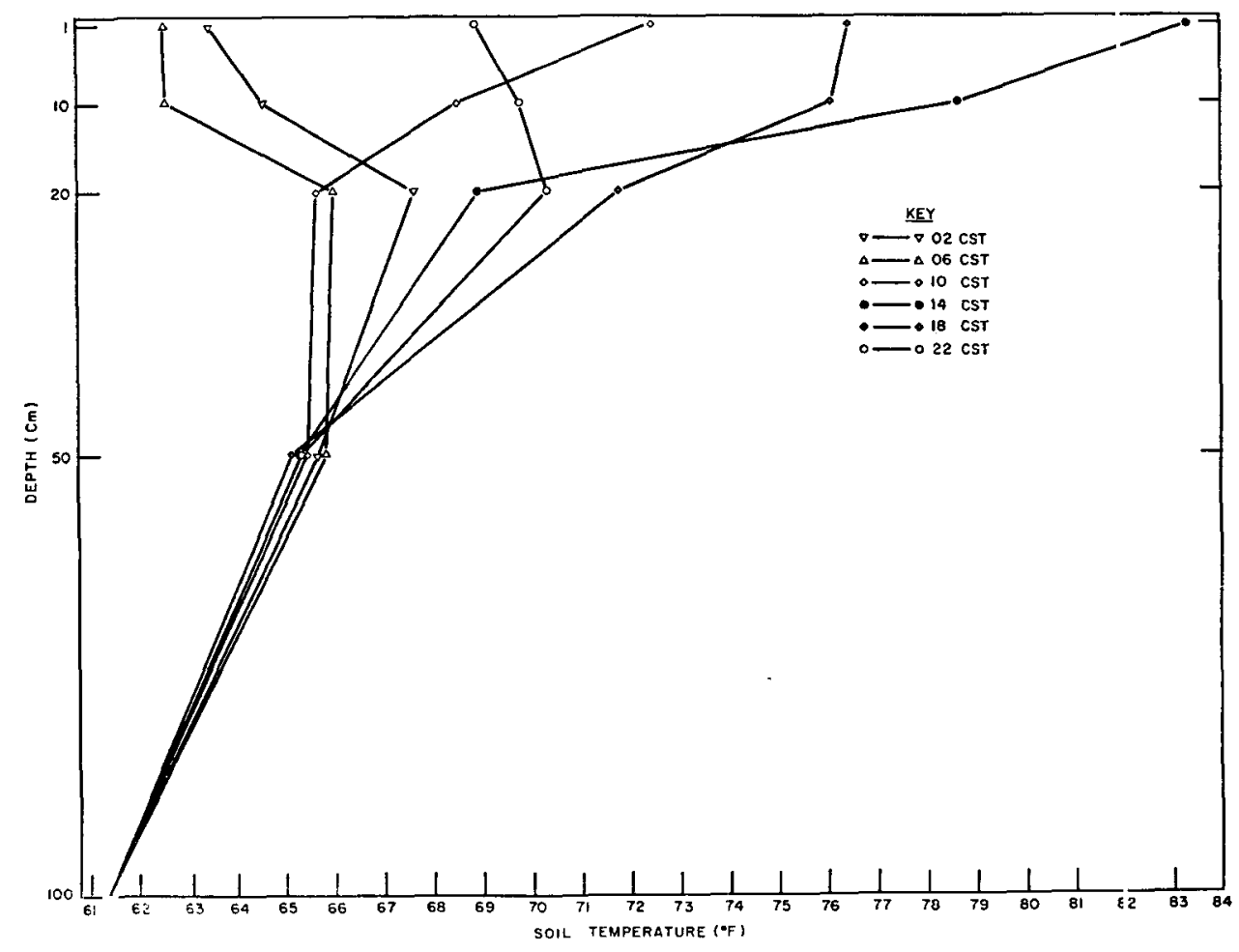

FIG. 4. Vertical temperature profiles in soil during the course of a typical summer day, 27 July 1955. 

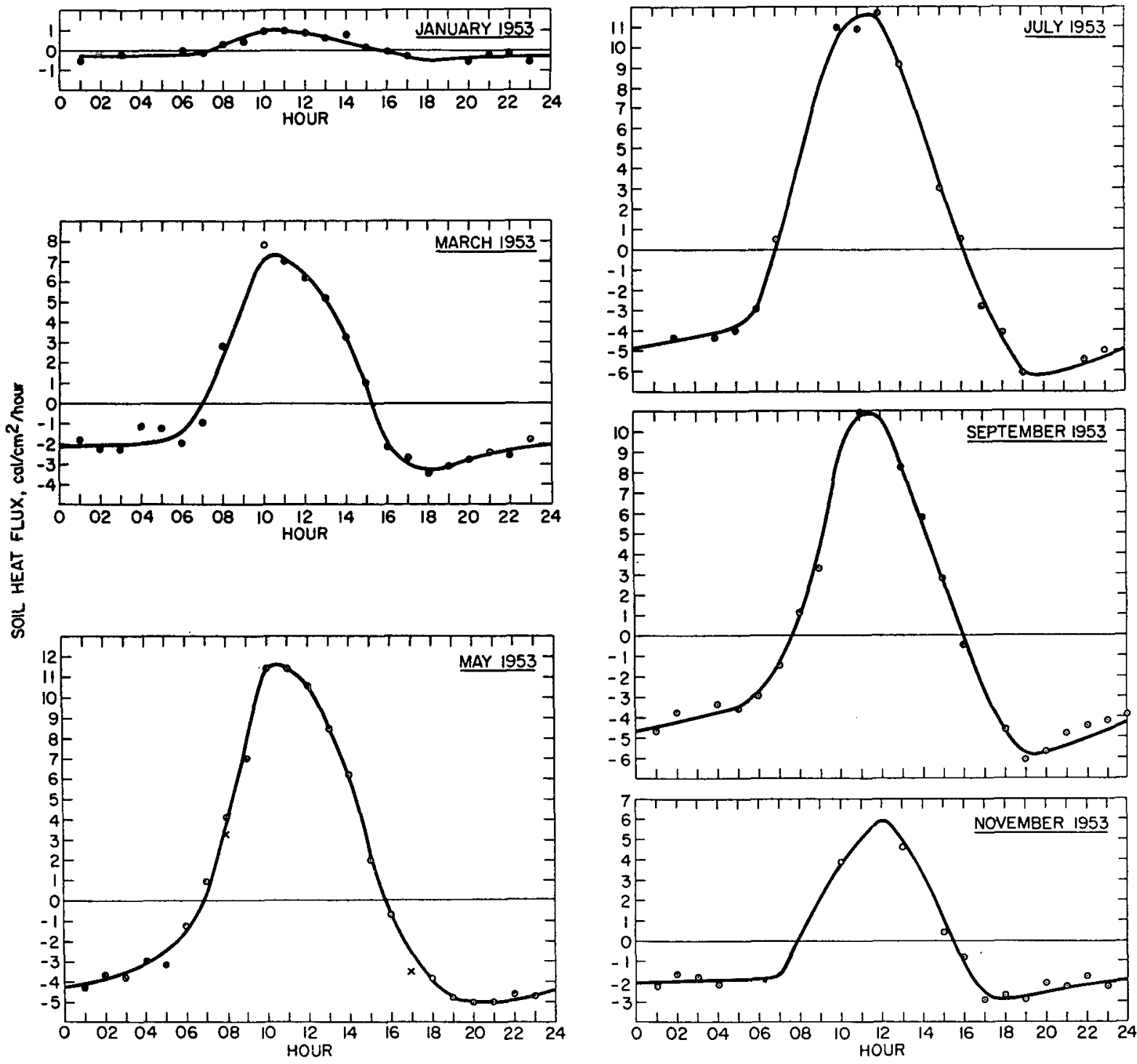

Frg. 5. Daily cycle of heat flux in the soil. Data based on average values of the 1-hr temperature change for the hour ending as indicated and on assumed values of soil heat capacity. Curves smoothed slightly.

20 per cent, since it was necessary to estimate values of heat capacity of the soil. Three field measurements of the moisture content and thermal capacity of the soil were made under very wet and very dry soil conditions. The extreme values of specific heat obtained, plus the rainfall record, were then used to estimate the heat capacity of soil used in the heat-exchange computations.

A parameter of considerable interest to meteorologists and climatologists is the amount of heat energy stored in the soil during the summer and then returned to the atmosphere during the following winter season. It was found that about 4500 to $5000 \mathrm{cal}$ were stored under each $\mathrm{cm}^{2}$ of turf during the warming part of the cycle.

A study of the annual cycle of heat flux into and out of the soil shows that the maximum heat exchanges are found in June and November, a month or two before the time of temperature extremes, in agreement with theory. The daily march of heat flux at the soil surface is quite similar to that of net radiation. The amplitude of the average daily heat exchange between the soil and air varied from $11.7 \mathrm{cal} \mathrm{cm}^{-2}$ in January (coldest month at Argonne) to $90.4 \mathrm{cal} \mathrm{cm}^{-2}$ in July (warmest month at Argonne).

Acknowledgments. The authors are grateful for the helpful comments and suggestions provided by Dr. John E. Rose, Director of the Radiological Physics Division, Argonne National Laboratory and Professor Horace R. Byers, Department of Geophysical Sciences, the University of Chicago. The assistance of Messers. Frank C. Kulhanek and Gunther A. Zerbe in obtaining the 
measurements and Mrs. Janet G. Daccardo and Miss Lee $M$. Cherven for the preparation of the figures and tables is sincerely appreciated.

\section{REFERENCES}

Budyko, M. I., 1958: Heat balance of the surface of the earth. Bull. World Meteor. Org., 7, 166-174.

Businger, J. A., and K. J. K. Buettner, 1961: Thermal contact coefficient (A term proposed for use in heat transfer). $J$. Meleor., 18, 422 .

Carson, J. E., 1963: Analysis of soil and air temperature by Fourier techniques. $J$. geophys. Res., 68, 2217-2232.

Haltiner, G. J., and F. L. Martin, 1957: Dynamical and physical meteorology. New York, McGraw-Hill, 470 pp.

Hann, J. von, and R. Süring, 1943: Lehrbuch der Meteorologie. 5th ed., Liepzig, Keller, $1092 \mathrm{pp}$.

Ingersoll, L. R., O. J. Zobel and A. C. Ingersoll, 1954: Heat conduction. Rev. ed., Madison, Univ. of Wisconsin Press, $325 \mathrm{pp}$.

Johnson, J. E., 1954: Physical meteorology. New York, Wiley and Sons, Inc., $393 \mathrm{pp}$.

Johnston, C. N., 1937: Heat conductivity of soil governs heat losses from heated oil lines. Petroleum Engr., 9, 41-48.

Kersten, M. S., 1949: Thermal properties of soils. Minn. Univ. Engr. Expt. Sta. Bull., 28, 228 pp.

Kühl, W., 1907: Der jährlichë Gang der Bodentemperatur in verschiedenen Klimaten. Ger. Beit. Geophys., 8, 499-564.

Kuhn, P. M., G. L. Darkow and V. E. Suomi, 1958: A mesoscale investigation of pre-tornado thermal environments. Bull. Amer. meteor. Soc., 39, 224-228.

Lettau, H. H., 1957 : Computation of heat budget constituents of the earth/air interface. Exploring the atmosphere's first mile, London, Pergamon Press, 305-327.
McCulloch, J. S. G., and H. L. Penman, 1956: Heat flow in the soil. 6th Internat. Cong. Soil Sci., v. B, Comm. I, 275-280.

Pasquill, F., 1949: Eddy diffusion of water vapour and heat near the ground. Proc. Roy. Soc. London, A198, 116-140.

Patten, H. E., 1909: Heat transference in soils. U. S. Dept. of Agr., Bur. Soils Bull., 59, 54 pp.

Pearce, D. C., and L. W. Gold, 1959: Observations of ground temperature and heat flow at Ottarva, Canada. J. geophys. Res., 64, 1293-1298.

Petterssen, S., and P. A. Calabrese, 1959: On some weather influences due to warming of the air by the Great Lakes in winter. J. Meteor., 16, 646-652.

Rider, N. E., 1955: An instrument for the continuous recording of soil temperature at a number of d'sths. Meteor. Mag., 84, 329-332.

- 1957 : A note on the physics of soil tempeature. Weather, 12, 241-246.

Saunders, W. E., 1960a: The clearance o: water fog following the arrival of a cloud sheet during the ight. Meteor. Mag., 89, 8-10.

$-1960 \mathrm{~b}$ : The clearance of frost following the arrival of a cloud sheet during the night. Meteor. Mag., 89, 45-51.

Schubert, J., 1904: Der Wärmeaustausch im festen Erdboden. Berlin, Springer, $30 \mathrm{pp}$.

Smagorinsky, J., 1953: The dynamical inf.uence of large-scale heat sources and sinks on the quasi-stationary mean motions of the atmosphere. Quart. J. R. meteor. Soc., 79, 342-366.

Smith, W. O., 1939: Thermal conductivities of moist soils. Proc. Soil Sci. Soc. Amer., 4, 32-40.

Vries, D. A. de, 1958: The thermal bshaviour of soils. Proc. Canberra. Sympos. Arid Zone Resear $/$, XI, Climatology and Microclimatology, 106-113. 